Pacific Journal of Mathematics

QUOTIENTS OF COMPLETE INTERSECTIONS BY $C^{*}$ 


\title{
QUOTIENTS OF COMPLETE INTERSECTIONS
}

\section{BY $C^{*}$ ACTIONS}

\author{
RICHARD RANDELL
}

We consider complete intersections $V$ in $C^{m}$ which have an isolated singularity at $\underline{0}$. When $V$ admits a $C^{*}$ action, one has the orbit space $V^{*}=V-\{0\} / C^{*}$. In this paper we determine when $V^{*}$ is a topological manifold, or in some cases, the precise dimension of the set $\Sigma$ along which $V^{*}$ is not a manifold. For proper actions we consider a natural complex structure on the space $V^{*}$ and determine some equivalences among $V^{*}$ for different $V$. Our methods are topological; the results are expressed numerically in terms of weighted degrees of the polynomials defining $V$.

1. Introduction. Let $f^{(1)}, \cdots, f^{(k)}$ be complex polynomials in $\underline{z}=\left(z_{1}, \cdots, z_{m}\right)$. Let $V\left(f^{(j)}\right)=\left\{\underline{z} \in C^{m} \mid f^{(j)}(\underline{z})=0\right\}$, and suppose that $V$ has an isolated singularity at $\underline{0}$ and is the complete intersection of the $V\left(f^{(j)}\right) ; \operatorname{dim}_{c} V=m-k$. We set $n=m-k$. Further suppose that there is an action of $C^{*}=C-\{0\}$ on $C^{m}$ of the form

$$
\sigma\left(t ; z_{1}, \cdots, z_{m}\right)=\left(t^{q_{1}} z_{1}, \cdots, t^{q_{m}} z_{m}\right)
$$

leaving $V$ invariant, with (i) $q_{i} \in Z, i=1, \cdots, m$ and (ii) g.c.d. $\left(q_{1}, \cdots, q_{m}\right)=1$. Such an action will be called a diagonal action of type $\left(q_{1}, \cdots, q_{m}\right)$. We assume that $V$ is not contained in any hyperplane so that (ii) implies the $C^{*}$ action is effective. Also, $q_{i} \neq 0$ implies that $\underline{0}$ is the only fixed point, while $q_{i}>0$ implies that the action is proper (i.e., the map $\psi: \boldsymbol{C}^{*} \times \boldsymbol{C}^{m} \rightarrow \boldsymbol{C}^{m} \times \boldsymbol{C}^{m}$ given by $\psi(t, \underline{z})=(\underline{z}, \sigma(t ; \underline{z}))$ is proper. $)$ We shall call such actions fixed-point free and proper, respectively.

Results of Holmann [5] show that for proper actions there is a unique complex structure on $V^{*}=V-\{0\} / C^{*}$ such that the orbit map is holomorphic. Later we will describe this structure in more detail.

By [10, Proposition (1.1.3)], any algebraic variety $V$ admitting a $C^{*}$ action given by a morphism of algebraic varieties may be embedded in some $\boldsymbol{C}^{m}$ so that the given action is induced by a diagonal action on $\boldsymbol{C}^{m}$. By the above, the action is proper and without fixed-points on $V-\{0\}$ precisely when $q_{i}>0$. Actions with $q_{i} \leqq 0$ are also of interest, as they arise when considering $C^{*}$ actions on versal deformations.

We next note that [10, Proposition (1.1.2)] allows us to assume that $V$ is defined by weighted (or quasi-) homogeneous polynomials. 
Recall that given an $m$-tuple $\underline{w}=\left(w_{1}, \cdots, w_{m}\right)$ of positive rationals we say that a polynomial is weighted homogeneous with weights $\underline{w}$ (or, $f$ is of type $\underline{w}$ ) if $a_{1} / w_{1}+\cdots+a_{m} / w_{m}=1$ for every monomial $\alpha z_{1}^{a_{1}} \cdots z_{m}^{a_{m}}$ of $f$. Write $w_{i}=u_{i} / v_{i},\left(u_{i}, v_{i}\right)=1$ and let $d=$ l.c.m. $\left(u_{1}, \cdots, u_{m}\right)$, $q_{i}=d / w_{i}$. Then

$$
f\left(t^{q_{1}} z_{1}, \cdots, t^{q_{m}} z_{m}\right)=t^{d} f\left(z_{1}, \cdots, z_{m}\right) .
$$

We call $d$ the polynomial degree of $f$ and $q_{i}, i=1, \cdots, m$ the coordinate degrees of $f$. The coordinate degrees are related to the $q_{i}$ of (1.1).

We may thus restate our situation: $V$ is a complete intersection of varieties $V\left(f^{(j)}\right), j=1, \cdots, k$, where $f^{(j)}$ is a weighted homogeneous polynomial with degree $d^{(j)}$ and coordinate degrees $q_{i}^{(j)}, i=$ $1, \cdots, m$. Furthermore, there are integers $\lambda^{(j)}$ with g.c.d. $\left(\lambda^{(1)}, \cdots\right.$, $\left.\lambda^{(k)}\right)=1$ so that $\left(q_{1}, \cdots, q_{m}\right)=\lambda^{(j)}\left(q_{1}^{(j)}, \cdots, q_{m}^{(j)}\right), j=1, \cdots, k$.

Since $V$ is a complete intersection we may conclude from work of Hamm [3] that $K=V \cap S^{2 m-1}$ is a $(2 n-1)$-dimensional manifold with an effective action of $S^{1} \subset C^{*}$. It is easily seen that $K^{*}=$ $K / S^{1}$ is homeomorphic to $V^{*}$, and we will often work with $K^{*}$.

In $\S 2$ we state some results on $S^{1}$ actions due to Neumann [8] which we use in $\S 4$, where we determine necessary conditions for $K^{*}$ to be a topological manifold. The most easily stated result is (with $q_{i} \neq 0$ ).

Corollary 4.4. Suppose $n>3$ and $K^{*}$ is a manifold. If the weights $\underline{w}^{(j)}$ are the same for all $j$, then the weights are integers, and $V$ is therefore equivariantly homeomorphic to a variety defined by Pham-Brieskorn polynomials.

In $\S 5$ we determine number-theoretic conditions sufficient to ensure that certain $K^{*}$ are manifolds, and in fact we determine precisely the dimension of the singular set. The final section studies the complex structure of $V^{*}$ if $q_{i}>0$. We show that $V^{*}$ is nonsingular as a complex space precisely when $K^{*}$ is a topological manifold. We also give a general criterion to determine when different $V$ yield biholomorphically equivalent $V^{*}$.

Many authors have studied these varieties. J. Milnor [7] was perhaps the first to notice that weighted homogeneous polynomials are topologically pleasant to work with. W. Neumann [8] considered many of the same problems for the Pham-Brieskorn polynomials $\Sigma z_{i}^{a} i$; we often use his techniques. G. Edmunds [2] gave an explicit embedding of $V^{*}$ into projective space. Finally, P. Orlik and P. Wagreich have contributed extensively to the study of varieties with $C^{*}$ actions $[10,11,12,13]$; it is a pleasure to thank them for 
many useful conversations and comments.

2. Slices and $S^{1}$ actions. It is convenient to work with the action of $S^{1}$ on $K$. In this section we briefly recall some language of slice diagrams (see Jänich [6] for more details) and state some results of Neumann [8] for quotients of linear actions of $S^{1}$ and finite cyclic groups.

Let $G$ be a compact Lie group. At every point $x$ of a $G$-manifold $X$ there is a slice $W_{x}$ transverse to the orbit $G(x)$ of $G$ at $x$. $W_{x}$ is a real vector space and the isotropy group $G_{x}=\{g \in G \mid g x=x\}$ acts effectively on $W_{x}$ via a representation $\sigma$. The slice theorem $[6,1.3]$ yields the following easy result.

THEOREM. Suppose $G$ is a compact Lie group acting effectively on a smooth manifold $X$. Then $X / G$ is a manifold if and only if $W_{x} / G_{x}$ is a manifold for every $x \in X$.

We will write $\left[G_{x}, \sigma\right]$ to indicate the action of $G_{x}$ on $W_{x}$ via $\sigma$, and we will call $\left[G_{x}, \sigma\right]$ the slice type at $x$. If $W_{x} / G_{x}$ is a manifold we say $\left[G_{x}, \sigma\right]$ has $Q M$.

In our situation we have an effective action of $S^{1}$ on $K$. Possible !sotropy groups are $\{1\}$, cyclic groups $Z_{q}$, and $S^{1}$ (possible only if some $\left.q_{i}=0\right)$. For $W=\boldsymbol{R}^{2}$ or $C$, we denote by $\sigma_{p}$ the real or complex representation of $S^{1}$ or $Z_{q}$ on $W$ given by

$$
\exp (i \theta) \longrightarrow \exp (i \theta p) \text {. }
$$

Every representation of $S^{1}$ or $Z_{q}$ as an isotropy group of the $S^{1}$ action on $K$ on the vector space $W_{x}$ is equivalent to one of the form $\sigma_{p_{1}} \oplus \cdots \oplus \sigma_{p_{r}} \oplus j$, where $j$ denotes a $j$-dimensional trivial representation.

Thus the following result of Neumann [8, Theorem 2.2] is crucial. As usual, we write $\left[G_{x}, \sigma_{p_{1}} \oplus \cdots \oplus \sigma_{p_{r}} \oplus j\right]$ for a slice with $G_{x}=Z_{q}$ or $S^{1}$ and indicated linear action of $G_{x}$ on $W_{x}$.

THEOREM (Neumann's criterion).

(i) Let g.c.d. $\left(p_{1}, \cdots, p_{r}, q\right)=1$ and let $\bar{p}_{i}=$ g.c.d. $\left(p_{1}, \cdots, \hat{p}_{i}\right.$, $\left.\cdots, p_{r}, q\right)$. Then for $r \geqq 1,\left[Z_{q}, \sigma_{p_{1}} \oplus \cdots \oplus \sigma_{p_{r}} \oplus j\right]$ has $Q M$ if and only if $\bar{p}_{1} \cdots \bar{p}_{r}=q$.

(ii) Let g.c.d. $\left(p_{1}, \cdots, p_{r}\right)=1$. Then $\left[S^{1}, \sigma_{p_{1}} \oplus \cdots \oplus \sigma_{p_{r}} \oplus j\right]$ has $Q M$ if and only if $r \leqq 2$.

Thus, $\left[\boldsymbol{Z}_{6}, \sigma_{2} \oplus \sigma_{3}\right]$ has $Q M$, while $\left[\boldsymbol{Z}_{6}, \sigma_{2} \oplus \sigma_{5}\right]$ does not.

3. The slice representation. From the preceding section it is 
clear that we must determine the various slice types of the action (1.1) on $S^{2 m-1}$ and $K$.

On $S^{2 m-1}$ the problem is trivial. At a point $\underline{z}$ with precisely the first $r$ coordinates nonzero the slice type is $\left[Z_{q},(r-1) \oplus \sigma_{q_{r+1}} \oplus \cdots \oplus \sigma_{q_{m}}\right]$, where $q=$ g.c.d. $\left(q_{1}, \cdots, q_{r}\right)$.

On $K$ the problem is slightly less trivial. Given an $r$-element subset $I_{r}$ of $\{1, \cdots, m\}$, we will write $q\left(I_{r}\right)=$ g.c.d. $\left\{q_{i}, i \in I_{r}\right\}$ and we will denote by $T\left(I_{r}\right)$ the slice type of $K$ at a point $\underline{z}$ whose nonzero coordinates are precisely those with subscripts in $I_{r} . O\left(I_{r}\right)$ will denote the orbit bundle of $T\left(I_{r}\right)$, that is, the set of the points of $K$ with slice type $T\left(I_{r}\right)$. It is easily seen that $\operatorname{dim}_{R} O\left(I_{r}\right) \geqq 2(r-k)-1$.

LEMMA 3.1. If $\operatorname{dim}_{\boldsymbol{R}} O\left(I_{r}\right)=2(r-k)-1$, then $T\left(I_{r}\right)=\left[Z_{q\left(I_{r}\right)}\right.$, $\left.(r-k-1) \oplus \sigma_{q_{r+1}} \oplus \cdots \oplus \sigma_{q_{m}}\right]$.

Proof. As in [8], this lemma is a consequence of the following: general fact: Suppose $Y$ is an invariant submanifold of $X$, and suppose that at some point $y \in Y$ the codimension of $Y$ in $X$ is the same as the codimension of the orbit bundle of $y$ in $Y$ in the orbit bundle of $y$ in $X$. Then the slice type of $y$ in $Y$ is the same, up to trivial factors, as the slice type of $x$ in $X$.

REMARK 3.2. In general, the slice representation at $\underline{z}$ in $K$ is a subrepresentation of the slice representation at $\underline{z}$ in $S^{2 m-1}$.

4. Bounds of the dimension of the singular set. Let $\Sigma$ be the subset of $K^{*}$ consisting of points where $K^{*}$ is not locally homeomorphic to $R^{2 n-2}$. We will call $\Sigma$ the singular set of $K^{*}$. Suppose $q_{i} \neq 0$ for all $i$. Recall that we have weights $w_{i}^{(j)}=u_{i}^{(j)} / v_{i}^{(j)}$, $\left(u_{i}^{(j)}, v_{i}^{(j)}\right)=1$, for $i=1, \cdots, m ; j=1, \cdots, k$. Let $t\left(I_{r}\right)=\operatorname{dim}_{C}(V \cap$ $\left\{z_{i}=0, i \notin I_{r}\right\}$.

THEOREM 4.1. Suppose $V$ is a complete intersection with isolated singularity at the origin, and suppose $V$ is defined by weighted homogeneous polynomials $f^{(j)}$ with weights $\underline{w}^{(j)}$. Further suppose that $V$ is invariant under a fixed-point free diagonal action of type $\left(q_{1}, \cdots, q_{m}\right)$. If (i) there are sets $I_{r} \subset\{1, \cdots, m\}$ and $J_{s} \subset\{1, \cdots, k\}$ with $r$ and $s$ elements respectively, so that some prime $p$ divides $v_{i}^{(j)}$ for $i \in I_{r}, j \in J_{s}$ and if (ii) $n-2(k-s)>3$, then

$$
\operatorname{dim}_{R} \Sigma \geqq 2\left(t\left(I_{r}\right)-1\right) \geqq 2\left(r^{r}-(k-s)-1\right) .
$$

Before proving this we state several corollaries and give some examples. As shown in [12] (and certainly to be expected), one is 
particularly interested in the question of when $K^{*}$ is a manifold.

CoRollary 4.2. Suppose $K^{*}$ is a manifold and $n>2 k+1$. Then for every $j \in\{1, \cdots, k\}$ and any $k$-element set $I_{k}$, one has g.c.d. $\left\{v_{i}^{(j)}, i \in I_{k}\right\}=1$.

Corollary 4.3. Suppose $n>3$. If there is a set $I_{r}$ so that $p$ divides $v_{i}^{(j)}$ for $i \in I_{r}, j=1, \cdots, k$ then $\operatorname{dim}_{R} \Sigma \geqq 2(r-1)$.

CoRollary 4.4. Suppose $n>3, K^{*}$ is a manifold, and the weights $\underline{w}^{(j)}$ are the same for all $j$. Then the weights are integers and $V$ is equivariantly homeomorphic to a variety defined by a complete intersection of Brieskorn varieties.

The last statement of 4.4 follows from the straightforward generalization of [10, Theorem 3.1.4].

These results are essentially the best possible: If $n=2, K^{*}$ is always a manifold. If $n=3$ we have the following

EXAmPLE 4.5. Let $n=3, k=1$, and define $V$ by $f\left(z_{1}, \cdots, z_{4}\right)=$ $z_{1}^{5}+z_{1} z_{2}^{6}+z_{3}^{3}+z_{3} z_{4}^{5}$. Then the weights are $(5,15 / 2,3,15 / 2)$, but one may compute slice types and apply Neumann's criterion to see that $K^{*}$ is a 4 -manifold.

EXAmple 4.6. The variety $V^{\prime}$ defined by the equations

$$
\begin{aligned}
& z_{1}^{4}+z_{2}^{6}+z_{3}^{20}+z_{4}^{28}+z_{5}^{44}+z_{6}^{52}=0 \\
& z_{1}^{3}+z_{1} z_{2}^{3}+z_{3}^{15}+z_{4}^{21}+z_{5}^{33}+z_{6}^{39}=0
\end{aligned}
$$

has $n=4, k=2$, and $w_{2}^{(2)}=9 / 2$. The reader may use Neumann's criterion and 3.1 to verify that $K^{*}$ is a manifold. (This will also follow from 5.3.) This example should be compared with 4.4.

Proof of 4.1. Suppose we have $I_{r}$ and $J_{s}$ satisfying (i) so that the first inequality of $(*)$ fails. Then we will show that (ii) also fails. In the course of doing this we will show that (i) implies $t\left(I_{r}\right) \geqq r-(k-s)$, giving the second inequality.

For convenience we will assume $I_{r}=\{1, \cdots, r\}$ and $J_{s}=\{1, \cdots, s\}$. Then for any monomial $\alpha z_{1}^{a_{1}} \cdots z_{r}^{a_{r}}$ of $f^{(j)}, j \in J_{s}$, one has

$$
a_{1} / w_{1}^{(j)}+\cdots+a_{r} / w_{r}^{(j)}=1 .
$$

But since $p$ divides $v_{i}^{(j)}, i \in I_{r}, j \in J_{s}$, the above equation implies that $p$ divides $u_{i}^{(j)}, i \in I_{r}, j \in J_{s}$. Since $\left(u_{i}^{(j)}, v_{i}^{(j)}\right)=1$ this is a contradiction, and no such monomial appears in $f^{(j)}, j \in J_{s}$. 
Therefore the set $S=\left\{\underline{z} \in C^{m} \mid z_{i}=0, i>r\right\}$ is contained in $\left\{f^{(1)}=\cdots=f^{(s)}=0\right\}$, so that $\operatorname{dim}_{c} V \cap S=t\left(I_{r}\right) \geqq r-(k-s)$.

Now let $S^{*}=S \cap K / S^{1}$. Then $\operatorname{dim}_{R} S^{*} \geqq 2\left(t\left(I_{r}\right)-1\right)$, so that if we let $\underline{z} \in S \cap K$ be a point with precisely the first $r$ coordinates nonzero, and if we assume that $\left(^{*}\right)$ fails, then the slice type at $\underline{z}$ must have $Q M$.

Let this slice type be $\left[Z_{q}, \sigma\right]$. Then $q=$ g.c.d. $\left(q_{1}, \cdots, q_{r}\right)$. Since $p$ divides $v_{i}^{(j)}, i=1, \cdots, r, p$ divides $q_{i}^{(j)}=d^{(j)} v_{i}^{(j)} u_{i}^{(j)}$, and thus $p$ divides $q$. Since $\sigma$ has $Q M$ it follows easily from Neumann's criterion and 3.2 that $p$ must divide at least $n-1$ of the $q_{i}$, say $p$ divides $q_{i}, i \in I_{n-1}$, where $I_{r} \subset I_{n-1}$. We may assume $I_{n}=\{1, \cdots, n-1\}$.

We next claim that in fact, $p$ divides $v_{i}^{(j)}, i \in I_{n-1}, j \in J_{s}$. By assumption $p$ divides $v_{i}^{(j)}, i \in I_{r} \subset I_{n-1}, j \in J_{s}$. For $i \in I_{n-1}, j \in J_{s}, p$ divides $q_{i}=\lambda^{(j)} d^{(j)} v_{i}^{(j)} / u_{i}^{(j)}$. If $p$ does not divide $v_{i}^{(j)}$, then $p$ divides $\lambda^{(j)} d^{(j)}$. This implies $p^{2}$ divides $q$ which in turn implies that $p^{2}$ divides $\lambda^{(j)} d^{(j)}$, etc. Thus $p$ divides $v_{i}^{(j)}, i \in I_{n-1}, j \in J_{s}$.

Now consider the $k \times m$ matrix $D=\left(d_{\alpha \beta}\right)$, where $d_{\alpha \beta}=\partial f^{(\alpha)} / \partial z_{\beta}$. We have seen that every monomial in $f^{(j)}, j \in J_{s}$, which contains a variable $z_{i}, i=1, \cdots, n-1$, must also contain some $z_{i}, i>n-1$. Let $P_{0}=\left\{z_{n}=\cdots=z_{m}=0\right\}$, and let $P=P_{0} \cap V$. Then $f^{(j)}(z)=0$, for $\underline{z} \in P_{0}, j \in J_{s}$, so $\operatorname{dim}_{c} P \geqq(n-1)-(k-s)$. On $P$ we clearly have $d_{\alpha \beta}=0,1 \leqq \alpha \leqq s, 1 \leqq \beta \leqq n-1$.

Of course, $V$ as a complete intersection is singular wherever $D$ has rank less than $k$. Let $D_{s}$ be the $s \times m$ matrix consisting of the first $s$ rows of $D$, and let $D_{s}^{\prime}$ be the $s \times(m-(n-1))=s \times(k+1)$ matrix consisting of the last $k+1$ columns of $D_{s}$. If the rank of $D_{s}^{\prime}$ is less than $s$ at any point $\underline{z}_{0}$ of $P$, then $V$ is singular at $\underline{z}_{0}$.

But $D_{s}^{\prime}$ will have rank less than $s$ if $k-s+2$ minors of size $s \times s$ vanish. Thus $V$ will be singular on a set of complex dimension at least $\operatorname{dim}_{c} P-(k-s+2) \geqq(n-1)-(k-s)-(k-s+2)=$ $n-2(k-s)-3$. Since $V$ has an isolated singularity, $n-2(k-s)-$ $3 \leqq 0$, contradicting (ii) and thus completing the proof.

We conclude this section with two trivial consequences of Neumann's criterion.

Proposition 4.7. Suppose $V$ is a complete intersection with isolated singularity and diagonal $C^{*}$ action, and suppose $q_{1}=\cdots=$ $q_{r}=0, q_{i} \neq 0, i>r$. If $K^{*}$ is a manifold, $n-\operatorname{dim}_{c} V \cap\left\{z_{i}=0\right.$, $i>r\} \leqq 2$.

Proof. The $S^{1}$ action on $K$ fixes $K \cap\left\{z_{i}=0, i>r\right\}$.

The next proposition is a topological analogue of a phenomenon noticed by G. Edmunds $[2, \S 5]$. 
Proposition 4.8. The real codimension of $\Sigma$ in $K$ is at least 4.

Proof. This is a trivial consequence of Neumann's criteria, as at any point the isotropy is $S^{1}$ or $Z_{q}$, and $K^{*}$ can fail to be a manifold at the point only if the slice representation has at least three or two nontrivial summands, respectively.

5. Totally complete intersections. In general one needs to know the form of the polynomials defining $V$ in order to determine the exact dimension of $\Sigma$. There is, however, one class of complete intersections for which a knowledge of the polynomial and coordinate degrees will suffice.

Definition 5.1. $\quad V^{n} \subset C^{m}$ is called a totally complete intersection if the intersection of $V$ with all coordinate subspaces of $C^{m}$ has minimal dimension.

An example is an intersection of Brieskorn varieties with suitable coefficients (see Hamm [4]). The complete intersection $V^{\prime}$ of 4.6 is another such example.

Definition 5.2. Given a complete intersection $V^{n}$ with diagonal $C^{*}$ action of type $\left(q_{1}, \cdots, q_{m}\right), q_{i} \neq 0$, we define $t_{i}=$ g.c.d. $\left(q_{1}, \cdots\right.$, $\left.\hat{q}_{i}, \cdots, q_{m}\right)$, and $s_{i}=q_{i} / t_{1} \cdots \hat{t}_{i} \cdots t_{m}$.

Since g.c.d. $\left(q_{1}, \cdots, q_{m}\right)=1$ we easily see that $\left(t_{i}, t_{j}\right)=1, i \neq j$, $s_{i} \in Z$, and $\left(s_{i}, t_{i}\right)=1, i=1, \cdots, m$. Let $\gamma$ be the largest integer such that there exist $\gamma$ of the $s_{i}$ with common divisor greater than one.

THEOREM 5.3. Suppose $V^{n} \subset C^{m}$ is a totally complete intersection with isolated singularity at $\underline{0}$ admitting a diagonal $\boldsymbol{C}^{*}$ action of type $\left(q_{1}, \cdots, q_{m}\right)$, with $q_{i} \neq 0, i=1, \cdots, m$. Then the real dimension of the singular set $\Sigma$ of the orbit space $V-\{0\} / C^{*}$ is $\max \{-1,2(n-m-1+\gamma)\}$.

Proof. We consider the associated $S^{1}$ action on $K$. At a point $\underline{z}$ of $K$ with precisely the first $\gamma$ coordinates nonzero, we have cyclic isotropy of order $q=$ g.c.d. $\left(q_{1}, \cdots, q_{\gamma}\right)$. By 3.1 , the slice representation is $\sigma=\sigma_{q_{\gamma+1}} \oplus \cdots \oplus \sigma_{q_{m}} \oplus(n+\gamma-m-1)$.

We now apply Neumann's criterion: $K^{*}$ will be a manifold if

$$
\prod_{s=1}^{m-r} \text { g.c.d. }\left(q_{1}, \cdots, q_{r}, q_{\gamma+1}, \cdots \hat{q}_{\gamma+s}, \cdots, q_{m}\right)=q
$$

(5.3.1) holds, by definition, if $t_{\gamma+1} \cdots t_{m}=$ g.c.d. $\left(q_{1}, \cdots, q_{\gamma}\right)$. The latter equation easily is seen to hold if and only if g.c.d. $\left(s_{1}, \cdots, s_{r}\right)=1$. 
Since the set $V \cap\left\{\underline{z} \mid\right.$ precisely $z_{1}, \cdots, z_{\gamma}$ are nonzero $\}$ has complex dimension $n-(m-\gamma)$, the result follows.

In particular, $K^{*}$ is a manifold if and only if no collection of $(k+1)$ of the $s_{i}$ has a common divisor.

For various applications (cf. [12, §4]) one wishes to construct $V$ with $C^{*}$ action so that $K^{*}$ is a manifold.

Proposition 5.4. Suppose integers $t_{i}, s_{i}, i=1, \cdots, m$ and $c_{j}$, $j=1, \cdots, k$ are given such that $\left(t_{i}, t_{j}\right)=1, i \neq j$, and $\left(s_{i}, t_{i}\right)=1$, for all $i$. Define $a_{i j}=\left(c_{j} t_{i}\right)\left[1 . c . m . ~\left(s_{1}, \cdots, s_{m}\right)\right] / s_{i}$. Then a totally complete intersection $V$ defined by the equations

$$
\sum_{i=1}^{m} \alpha_{i j} z_{i}^{a_{i j}}=0, \quad j=1, \cdots, k
$$

has a $C^{*}$ action. The associated $K^{*}$ is a manifold if and only if no $k+1$ of the numbers $s_{1}, \cdots, s_{m}$ have a common divisor.

Proof. This follows from 5.3 and easy computations which yield

$$
\begin{aligned}
& d^{(j)}=c_{j}\left[\text { l.c.m. }\left(s_{1}, \cdots, s_{m}\right)\right] t_{1} \cdots t_{m} \\
& q_{i}=t_{1} \cdots t_{i-1} s_{i} t_{i+1} \cdots t_{m} .
\end{aligned}
$$

Neumann proved 5.4 for $k=1$. We should emphasize that 5.3 does not depend on the polynomials themselves, but only on the polynomial and coordinate degrees.

6. The complex spaces $V^{*}$. We now change our viewpoint somewhat and require $q_{i}>0, i=1, \cdots, m$, so that the action (1.1) is proper.

We give $V^{*}=V-\{0\} / C^{*}$ a complex structure as in Brieskorn and Van de Ven [1]: Define a holomorphic operation of $C$ on $V-\{0\}$ by

$$
t\left(z_{1}, \cdots, z_{m}\right)=\left(\exp \left(t q_{1}\right) z_{1}, \cdots, \exp \left(t q_{m}\right) z_{m}\right) .
$$

Notice that an orbit of the $C$ action on $V$ intersects $K$ in an orbit of the $S^{1}$ action on $K$. In fact the imaginary axis from 0 to $2 \pi i$ moves any point of $K$ through its $S^{1}$ orbit. Thus $V-\{0\} / C \cong V^{*} \cong K^{*}$.

Consider $\boldsymbol{Z} \subset \boldsymbol{C}$ as an additive subgroup and let $H=V-\{0\} / Z$. It is easily seen that $H \cong K \times S^{1}$. Let $\Gamma$ be the discrete subgroup of $C$ generated by 1 and $2 \pi i$. The torus $T=C / \Gamma$ acts on $H$ by (6.0.1), and by results of Holmann [5], $H / T$ is a complex space homeomorphic to $V^{*}$ or $K^{*}$.

TheOREM 6.1 (Neumann [8] for Brieskorn varieties). Suppose $V$ is a complete intersection with proper diagonal $C^{*}$ action, and 
suppose $V$ has an isolated singularity at $\underline{0}$. Then $K^{*}$ is a manifold if and only if the complex structure on $V^{*}$ is nonsingular.

Proof. A theorem of Prill [14] asserts that the complex structure is nonsingular if and only if the isotropy group at every point $p$ is generated by elements of $T$ with complex codimension one fixedpoint sets passing through $p$.

Let $K$ denote the intersection of $V$ with the unit sphere and let $\underline{z} \in K, \theta \in S^{1}$. Then the $T$ action on $H$ is given by $(a, b)(\underline{z}, \theta)=$ $(b(\underline{z}), a(\theta))$, where $(a, b)$ are coordinates of $T$ in the direction of 1 and $2 \pi i$. Clearly the isotropy at $(\underline{z}, \theta) \in H$ is the same as the isotropy of the $S^{1} \subset T, S^{1}=\{(0,2 \pi i \theta) \mid 0 \leqq \theta<1\}$ and this in turn is the same as the isotropy of the $S^{1}$ action on $K$ at $\underline{z}$.

The result then follows by direct comparison of the criterion of Neumann for $K^{*}$ to be a manifold with the above criterion of Prill.

We now generalize the concept of the cone over $V,[10]$. We no longer assume that $V$ is a complete intersection or has an isolated singularity at $\underline{0}$. We do of course continue to assume that $V$ is invariant under a proper diagonal $\boldsymbol{C}^{*}$ action.

Definition 6.2. Suppose $V$ is defined by polynomials $f^{(j)}=$ $\Sigma \alpha z_{1}^{a_{1}} \cdots z_{m}^{a_{m}}$. The variety $V_{0}$ defined by $g^{(j)}=\Sigma \alpha z_{1}^{a_{1} r_{1}} \cdots z_{m}^{a_{m} r_{m}}$ is called the weighted cone over $V$ with weights $\left(r_{1}, \cdots, r_{m}\right) \in\left(Z^{+}\right)^{m}$.

Note that $\phi\left(z_{1}, \cdots, z_{m}\right)=\left(z_{1}^{r_{1}}, \cdots, z_{m}^{r_{m}}\right)$ defines a map $\phi: V_{0} \rightarrow V$, and that $\phi$ has degree $r_{1} \cdots r_{m}$ so long as $V$ is contained in no coordinate hyperplane. In [10], the weighted cone with weights $\left(q_{1}, \cdots, q_{m}\right)$ was called simply the cone over $V$. We will call this special case the minimal homogeneous cone over $V . V_{0}$ admits a proper diagonal $C^{*}$ action which commutes with $\phi$, so that one obtains a map $\psi: V_{0}^{*} \rightarrow V^{*}$ of complex spaces. Thus if $V_{0}$ is the minimal homogeneous cone, $V^{*}$ is branch covered by a projective variety.

We next ask for the degree of $\psi$, and in particular, when is $\psi$ biholomorphic?

THEOREM 6.3. Let $V_{0}$ be a variety with proper diagonal $C^{*}$ action of type $\left(q_{1}, \cdots, q_{m}\right)$. Define $t_{i}=$ g.c.d. $\left(q_{1}, \cdots, \hat{q}_{i}, \cdots, q_{m}\right)$. Suppose $V_{0}$ is the weighted cone over $V$ of type $\left(r_{1}, \cdots, r_{m}\right)$, and define $e_{i}=$ g.c.d. $\left(r_{i}, t_{i}\right)$. Then the degree of $\psi: V_{0}^{*} \rightarrow V^{*}$ is $r_{1} \cdots r_{m} / e_{1} \cdots e_{m}$.

Proof. The finite group $G=Z_{r_{1}} \oplus \cdots \oplus \boldsymbol{Z}_{r_{m}}$ acts on $V_{0}$ and $V_{0} / G \cong V$. Similarly, $G$ acts on $V_{0}^{*}$, and $V_{0}^{*} / G=V^{*}$. However, the latter action is not effective in general. Setting $G^{\prime}=\left\{g \in G \mid g z^{*}=z^{*}\right.$, for all $\left.z^{*} \in V_{0}^{*}\right\}$, we must show that the order of $G^{\prime}$ is $e_{1} \cdots e_{m}$.

Let $\beta_{i}$ generate $Z_{r_{i}}$, so that $\beta^{(i)}=\left(1, \cdots, \beta_{i}, \cdots, 1\right) \in G$ acts on 
$V_{0}$ by fixing all coordinates except the $i$ th, which is multiplied by $\exp \left(2 \pi i / r_{i}\right)$. Let $\gamma_{i}=\beta_{i}^{r_{i} / e_{i}}$ and $\gamma^{(i)}=\left(1, \cdots, \gamma_{i}, \cdots, 1\right)$. We claim that $G^{\prime}$ is generated by the $\gamma^{(i)}$.

We show first that $\gamma^{(i)} z^{*}=z^{*}$. That is, we show that $\underline{z}$ and $\gamma^{(i)}(\underline{z})$ are in the same orbit of the $C^{*}$ action on $V_{0}$. Let $\zeta=$ $\exp \left(2 \pi i / e_{i}\right)$. Then $\zeta\left(z_{1}, \cdots, z_{m}\right)=\left(z_{1}, \cdots, z_{i-1}, \zeta^{q_{i}} z_{i}, z_{i+1}, \cdots, z_{m}\right)$, since $\zeta^{q_{j}}=\exp \left(2 \pi i q_{j} / e_{i}\right)=1$ because $e_{i}$ divides $t_{i}$ and $t_{i}$ divides $q_{j}, i \neq j$. Further, since g.c.d. $\left(q_{i}, e_{i}\right)=1$, some power of $\zeta$ maps $\underline{z}$ to $\gamma^{(i)}(\underline{z})$. Thus $\gamma^{(i)} z^{*}=z^{*}$.

A similar argument shows that any element of $G^{\prime}$ must be a product of $\gamma^{(i)}$, and the result follows.

COROLLARY 6.4. Let $V_{0}$ be the minimal homogeneous cone over V. Then $\operatorname{deg} \phi=\operatorname{deg} \psi=q_{1} \cdots q_{m}$.

Proof. g.c.d. $\left(q_{i}, t_{i}\right)=1, i=1, \cdots, m$.

COROLLARY 6.5. $\psi$ is biholomorphic if and only if $r_{i}$ divides $t_{i}, i=1, \cdots, m$.

This was proved by Neumann for Brieskorn varieties.

REMARK 6.6. The restriction of $\psi$ to coordinate hyperplanes may not have the expected degree. For instance, if $V_{0}$ is defined by $z_{1}^{6}+z_{2}^{6}+z_{3}^{6}$ and $V$ is defined by $z_{1}^{2}+z_{2}^{3}+z_{3}^{6}$, deg $\psi=6$ but $\left.\operatorname{deg} \psi\right|_{z_{1}=0}=2$, since the restricted $C^{*}$ action is not effective.

Corollary 6.5 shows that one cannot obtain biholomorphic complex spaces by considering weighted cones between $V$ and the minimal homogeneous cone. One can obtain biholomorphic complex spaces by dividing the exponents of the defining polynomial by $t_{i}$, assuming that such division yields a polynomial. Our final result shows that one does get a polynomial.

Proposition 6.7. Suppose $V$ is a hypersurface with an isolated singularity at $\underline{0}$ and suppose $V$ admits a proper diagonal $C^{*}$ action of type $\left(q_{1}, \cdots, q_{m}\right)$. If $V$ is defined by $f$, with

$$
f\left(z_{1}, \cdots, z_{m}\right)=\Sigma \alpha z_{1}^{a_{1}} \cdots z_{m}^{a_{m}} .
$$

Then $t_{i}$ divides $a_{i}$ for every monomial of $f$.

Proof. Let $z_{1}^{a_{1}} \cdots z_{m}^{a_{m}}$ be a monomial of $f$, with polynomial degree $d$. Then, since $w_{i}=d / q_{i}$, we have 


$$
a_{1} q_{1}+\cdots+a_{m} q_{m}=d
$$

Since $t_{i}$ divides $q_{j}$ for $i \neq j$, and $\left(t_{i}, q_{i}\right)=1$ we see that $t_{i}$ divides $a_{i}$ if and only if $t_{i}$ divides $d$. Since $t_{i}$ divides $q_{j}, t_{i}$ divides $d v_{j} / u_{j}$, so if $t_{i}$ does not divide $d, t_{i}$ must divide $v_{j}, i \neq j$. Then, as in the proof of 4.1, we see that $f$ has at most 2 variables, if the singularity is isolated. So we are done for $m>2$. For $m=1$ the result is trivial, and for $m=2$ it may be checked by direct computation.

EXAMPLE. $\quad z_{1}^{a_{1}}+z_{1} z_{2}^{a_{2}}+z_{2} z_{3}^{a_{2}}$, with $\left(\alpha_{1}-1, \alpha_{2}\right)=1, \quad\left(\alpha_{2} \alpha_{3}, a_{1} \alpha_{2}-\right.$ $\left.a_{1}+1\right)=1$. The weights, in reduced form, are

$$
w_{1}=a_{1}, \quad w_{2}=a_{1} a_{2} /\left(a_{1}-1\right), \quad w_{3}=a_{1} a_{2} a_{3} /\left(a_{1} a_{2}-a_{1}+1\right) .
$$

Thus, $q_{1}=a_{2} a_{3}, q_{2}=a_{3}\left(a_{1}-1\right), q_{3}=a_{1} a_{2}-a_{1}+1$. Then

$$
\begin{aligned}
& t_{1}=\text { g.c.d. }\left(\left(a_{1}-1\right),\left(a_{1} a_{2}-a_{1}+1\right)\right)=1 \\
& t_{2}=\text { g.c.d. }\left(a_{2} a_{3}, a_{1} a_{2}-a_{1}+1\right)=1 \\
& t_{3}=\text { g.c.d. }\left(a_{2} a_{3}, a_{3}\left(a_{1}-1\right)\right)=a_{3} .
\end{aligned}
$$

\section{REFERENCES}

1. E. Brieskorn and A. Van de Ven, Some complex structures on products of homotopy spheres, Topology, 7 (1968), 389-393.

2. G. Edmunds, On the orbit space of a weighted homogeneous hypersurface, Proc. London Math. Soc., 29 (1974), 684-698.

3. H. Hamm, Lokale topologische Eigenschaften komplexer Räume, Math. Ann., 191 (1971), 235-252.

4. - Exotische Sphären als Umgebungsränder in speziellen komplexen Räumen, Math. Ann., 197 (1972), 44-56.

5. H. Holmann, Quotienträume komplexer Mannigfaltigkeiten nach komplexen Liescher Automorphismengruppen, Math. Ann., 139 (1960), 383-402.

6. K. Jänich, Differenzierbare G-Mannigfaltigkeiten, Springer lecture notes in math., No. 59, 1968.

7. J. Milnor, Singular Points of Complex Hypersurfaces, Princeton University Press, 1968.

8. W. Neumann, $S^{1}$-actions and the $\alpha$-invariant of their involutions, Bonn. Math. Schr. 44, 1970.

9. P. Orlik and R. Randell, The classification and monodromy of weighted homogeneous singularities, preprint.

10. P. Orlik and P. Wagreich, Isolated singularities of algebraic surfaces with $C^{*}$ action, Ann. of Math., 93 (1971), 205-228.

11. Singularities of algebraic surfaces with $C^{*}$ action, Math. Ann., 193 (1971), 121-135.

12. - Seifert n-manifolds, Invent. Math., 28 (1975), 137-159.

13. - Algebraic surfaces with $k^{*}$-action, Acta Math., 138 (1977), 43-81.

14. D. Prill, Local classification of quotients of complex manifolds by discontinuous groups, Duke Math. J., 34 (1967), 375-386.

Received February 14, 1977.

The Institute for Advanced Study

PRINCETON, NJ 08540 



\section{PACIFIC JOURNAL OF MATHEMATICS}

\section{EDITORS}

RICHARD ARENS (Managing Editor)

University of California

Los Angeles, California 90024

C. W. Curtis

University of Oregon

Eugene, OR 97403

C. C. MOORE

University of California

Berkeley, CA 94720

\section{J. DUGUNDJI}

Department of Mathematics University of Southern California Los Angeles, California 90007

R. Finn AND J. Milgram Stanford University Stanford, California 94305

ASSOCIATE EDITORS
E. F. BECKENBACH

B. H. NeUMANN

F. WOLF

K. YoshidA

\section{SUPPORTING INSTITUTIONS}

UNIVERSITY OF BRITISH COLUMBIA UNIVERSITY OF SOUTHERN CALIFORNIA CALIFORNIA INSTITUTE OF TECHNOLOGY STANFORD UNIVERSITY UNIVERSITY OF CALIFORNIA MONTANA STATE UNIVERSITY UNIVERSITY OF TOKYO UNIVERSITY OF NEVADA, RENO UNIVERSITY OF UTAH NEW MEXICO STATE UNIVERSITY OREGON STATE UNIVERSITY UNIVERSITY OF OREGON

WASHINGTON STATE UNIVERSITY UNIVERSITY OF WASHINGTON OSAKA UNIVERSITY 


\section{Pacific Journal of Mathematics}

\section{Vol. 74, No. $1 \quad$ May, 1978}

Gerald Arthur Anderson, Computation of the surgery obstruction groups

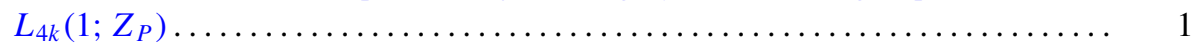

R. K. Beatson, The degree of monotone approximation ................ 5

Sterling K. Berberian, The character space of the algebra of regulated functions . . . 15

Douglas Michael Campbell and Jack Wayne Lamoreaux, Continua in the plane with

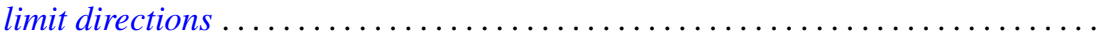

R. J. Duffin, Algorithms for localizing roots of a polynomial and the Pisot

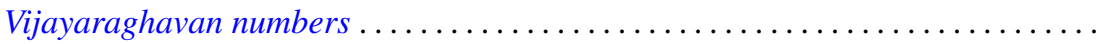

Alessandro Figà-Talamanca and Massimo A. Picardello, Functions that operate on

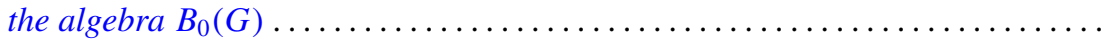

John Erik Fornaess, Biholomorphic mappings between weakly pseudoconvex

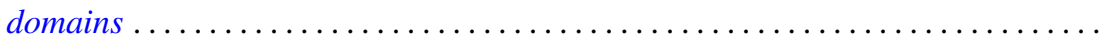

Andrzej Granas, Ronald Bernard Guenther and John Walter Lee, On a theorem of S.

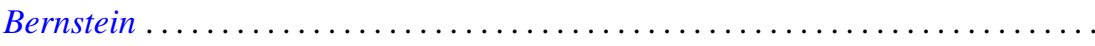

Jerry Grossman, On groups with specified lower central series quotients . .........

William H. Julian, Ray Mines, III and Fred Richman, Algebraic numbers, a constructive development . . . . . . . . . . . . . . . . . . . . . . .

Surjit Singh Khurana, A note on Radon-Nikodým theorem for finitely additive

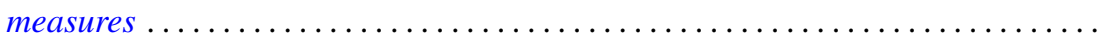

Garo K. Kiremidjian, A Nash-Moser-type implicit function theorem and nonlinear

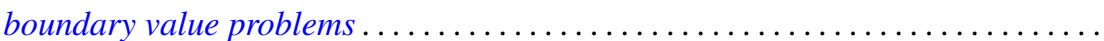

Ronald Jacob Leach, Coefficient estimates for certain multivalent functions ....

John Alan MacBain, Local and global bifurcation from normal eigenvalues. II . . 133

James A. MacDougall and Lowell G. Sweet, Three dimensional homogeneous algebras...

John Rowlay Martin, Fixed point sets of Peano continua ......

R. Daniel Mauldin, The boundedness of the Cantor-Bendixson order of some analytic sets...

Richard C. Metzler, Uniqueness of extensions of positive linear functions ..

Rodney V. Nillsen, Moment sequences obtained from restricted powers . .

Keiji Nishioka, Transcendental constants over the coefficient fields in differential elliptic function fields...

Gabriel Michael Miller Obi, An algebraic closed graph theorem

Richard Cranston Randell, Quotients of complete intersections by $\mathbf{C}^{*}$ actions . . 221

Bruce Reznick, Banach spaces which satisfy linear identities . .

Bennett Setzer, Elliptic curves over complex quadratic fields...

Arne Stray, A scheme for approximating bounded analytic functions on certain subsets of the unit disc.

Nicholas Th. Varopoulos, A remark on functions of bounded mean oscillation and bounded harmonic functions. Addendum to: "BMO functions and the

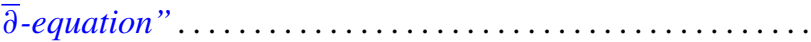

Charles Irvin Vinsonhaler, Torsion free abelian groups quasi-projective over their

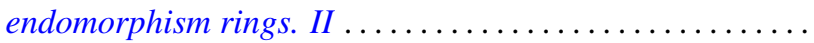

Thomas R. Wolf, Characters of $p^{\prime}$-degree in solvable groups ... 